\title{
Bed Rest in Pregnancy and Its Related Complications: Is It Needed?
}

\section{Maninder Ahuja}

\begin{abstract}
For centuries it is common myth to advice bed rest during pregnancy. At the least pretext bed rest is advised by family members and by health care providers also. But review of literature and RCT shows that in reality bed rest does not alter the course of pregnancy in various complications. Role of bed rest has been examined in singleton, twin and triplet pregnancies but was not found useful.

Prolonged bed rest is rather harmful as it causes increased calcium excretion, loss of muscle mass, financial loss and increased psychological rest for the pregnant woman and her family.

Moderate exercise is advisable throughout pregnancy to maintain tone of muscles and range of movements.

So till we have more proof we should be cautious in advising pregnant patients about bed rest. It can be limited activity where we feel it is not advisable to overexert her.
\end{abstract}

Keywords: Exercise, Pregnancy, Bed rest, Multiple pregnancy, Triplet pregnancy prolonged bed rest.

How to cite this article: Ahuja M. Bed Rest in P regnancy and Its Related Complications: Is It Needed?. J South Asian Feder Obst Gynae 2012;4(3):147-150.

\section{Source of support $\mathrm{Nil}$ \\ Conflict of interest: $\mathrm{Nil}$}

\section{INTRODUCTION}

Historically, bed rest has been prescribed in pregnancy for problems of threatened abortion, premature rupture of membranes, IUGR, PIH, placenta previa, pregnancy with hypertension, pregnancy with heart disease, $\mathrm{BOH}$ and sometimes even for vague pain abdomen in pregnancy. In some families, bed rest is advised as such without any complications in pregnancy. But, scientific data, point to just the opposite direction now not rest for normal pregnancy but rather exercise in pregnancy is prescribed and it is in the same way as historically earlier bed rest was prescribed for heart disease and now exercise and not bed rest is being prescribed for heart patients.

W ith limitation of beds available in our country and more couples living in nuclear families in our country it becomes relevant to find out, if bed rest has any role to play in pregnancyrelated complications.

So, with this idea to find out whether bed rest in pregnancy and its related complications helps or rather is harmful, review of literature was done to find out role of bed rest in pregnancy, for a better care for the patients, as unnecessary bed rest not only increases financial burden on the family but is rather cause of more psychological problems for patients and her family.

We would review literature regarding role of bed rest in pregnancy-related complications.

\section{Bed Rest in Singleton Pregnancies for Preventing Preterm Birth}

\section{Cochrane Rev Abstract. 2006; (02006 \\ The Cochrane Collaboration}

Study: Total of 1266 women were studied in this trial. B ut, this trial has uncertain methodological quality due to lack of reporting. Four hundred and thirty-two women were prescribed bed rest at home and a total of 834 women received a placebo (412) or no intervention (422). Preterm birth before 37 weeks was similar in both groups $(7.9 \%$ in the intervention group vs $8.5 \%$ in the control group) and the relative risk was 0.92 with a $95 \%$ confidence interval from 0.62 to 1.37 . No other results were available.

\section{Conclusion}

There is no evidence, either supporting or refuting the use of bed rest at home or in hospital, to prevent preterm birth. A lthough bed rest in hospital or at home is widely used as the first step of treatment, there is no evidence that this practice could be beneficial. Due to the potential adverse effects that bed rest could have on women and their families, and the increased costs for the healthcare system, clinicians should not routinely advise women to rest in bed to prevent preterm birth. Potential benefits and harms should be discussed with women facing an increased risk of preterm birth. A ppropriate research is mandatory. Future trials should evaluate both the effectiveness of bed rest and the effectiveness of the prescription of bed rest, to prevent preterm birth.

\section{HOSPITALIZATION AND BED REST IN MULTIPLE PREGNANCIES}

\section{Background}

Women and infants of a multiple pregnancy are recognized to be at increased risk of adverse outcome when compared with singletons. The greatest risk to infants of a multiple pregnancy is being born preterm, with preterm birth, defined as birth less than 37 weeks gestation and very preterm birth less than 32 weeks gestation. The preterm birth rate less than 37 w eeks for women with a singleton pregnancy is $6.3 \%$ vs $97 \%$ for women with a triplet pregnancy ${ }^{1}$ the mean gestational age of birth for infants of a triplet pregnancy being 31.9 weeks, with $39.3 \%$ if infants born before 32 weeks gestation, and a further $57.7 \%$ between 32 and 36 weeks gestation. ${ }^{1}$ Infants of a triplet pregnancy are at increased risk of poor intrauterine growth; with the mean birth weight of a triplet infant being $1668 \mathrm{gm}$, compared with $3398 \mathrm{gm}$ in singleton infants. ${ }^{1} \mathrm{~A}$ t birth, $15.9 \%$ of triplet infants weigh less than $1000 \mathrm{gm}, 35.9 \%$ less than $1500 \mathrm{gm}$ and $92.9 \%$ less than $2500 \mathrm{gm} .{ }^{1}$ Infants of a higher order multiple pregnancy are at increased risk of perinatal death, 
with a rate of 53.0/1000, almost 7 times greater than that observed in singletons. ${ }^{1}$

\section{HOSPITALIZATION FOR BED REST FOR WOMEN WITH A TRIPLET PREGNANCY}

\section{Background}

This abandoned randomized controlled trial assessed the effects of hospitalization from 24 to 30 weeks gestation for women with a triplet pregnancy on the risk of preterm birth.

\section{Results}

Seven women with a triplet pregnancy were recruited to the trial with three randomized to the hospitalization group and four to the control group. There were no statistically significant differences between the two groups for the primary outcomes birth before 37 weeks ( $3 / 3$ hospitalization group vs $4 / 4$ control group; relative risk (RR) not estimable), birth before 34 weeks (3/3 hospitalization group vs 2/4 control group; RR $2.0095 \%$ confidence intervals $(\mathrm{CI})$ 0.75-5.33) and pregnancy-induced hypertension (1/3 hospitalization group vs $1 / 4$ control group; RR 1.33 95\% CI 0.13-13.74).

When the results of this trial were incorporated into a metaanalysis with the previous randomized controlled trial assessing hospital ization and bed rest for women with a triplet pregnancy, (total sample size 26 women and 78 infants), there were no statistically significant differences identified between the two groups.

\section{Conclusion}

The results of this trial and meta-analysis suggest no benefit of routine hospitalization and bed rest for women with a triplet pregnancy to reduce the risk of preterm birth. The adoption or continuation of a policy of routine hospital ization and bed rest for women with an uncomplicated triplet pregnancy cannot be recommended.

\section{HOSPITALIZATION AND BED REST IN TWIN PREGNANCY}

Hospital admission has been advocated in the past for women with a twin pregnancy, as a means of reducing the risk of preterm birth and improving fetal growth. However, the Cochrane Systematic Review assessing the role of hospital ization and bed rest for women with an uncomplicated twin pregnancy has found the practice to beassociated with an increase in the risk of preterm birth, and should not be offered as part of routine care

[Crowther, C. The Cochrane Library. Chichester, UK : ] ohn Wiley \& Sons L td; 2004. H ospitalization for bed rest in multiple pregnancies (Cochrane Review)].

\section{BED REST WITH OR WITHOUT HOSPITALIZATION FOR HYPERTENSION DURING PREGNANCY}

\section{Main Results}

Four small trials (449 women) were included. Three were of good qual ity. T wo trials (145 women) compared strict bed rest with some rest, in hospital, for women with proteinuric hypertension. There was insufficient evidence to demonstrate any differences between the groups for reported outcomes. Two trials (304 women) compared some bed rest in hospital with routine activity at home for nonproteinuric hypertension. There was reduced risk of severe hypertension (1 trial, 218 women; RR $0.58,95 \% \mathrm{Cl} 0.38$ to 0.89 ) and a borderline reduction in risk of preterm birth ( 1 trial, 218 women; RR $0.53, \mathrm{Cl} 0.29$ to 0.99 ) with some rest compared to normal activity. M ore women in the bed rest group opted not to have the same management in future pregnancies, if the choice were given ( 1 trial, 86 women; RR $3.00,95 \% \mathrm{Cl} 1.43$ to 6.31 ). There were no significant differences for any other outcomes.

\section{Conclusion}

Few randomized trials have evaluated rest for women with hypertension during pregnancy, and important information on side-effects and cost implication is missing from available trials. A lthough one small trial suggests that some bed rest may be associated with reduced risk of severe hypertension and preterm birth, these findings need to be confirmed in larger trials. At present, there is insufficient evidence to provide clear guidance for clinical practice. Therefore, bed rest should not be recommended routinely for hypertension in pregnancy, especially since more women appear to prefer unrestricted activity, if the choice were given.

(Cochrane Rev A bstract. 2006; @2006 The Cochrane Collaboration)

\section{BED REST DURING PREGNANCY FOR PREVENTING MISCARRIAGE}

\section{Background}

M iscarriage is pregnancy loss before 23 weeks of gestational age and it happens in 10 to $15 \%$ of pregnancies depending on maternal age and parity. It is associated with chromosomal defects in about a half or two-thirds of cases. M any interventions have been used to prevent miscarriage but bed rest is probably the most commonly prescribed especially in cases of threatened miscarriage and history of previous miscarriage. Since, the etiology of miscarriage in most of the cases is not related to an excess of activity, it is unlikely that bed rest could be an effective strategy to reduce spontaneous miscarriage.

\section{Objectives}

To evaluate the effect of prescription of bed rest during pregnancy to prevent miscarriage in women at high-risk of miscarriage.

\section{Main Results}

Only two studies including 84 women were identified. There was no statistically significant difference in the risk of miscarriage in the bed rest group vs the no bed rest group (placebo or other treatment) [relative risk (RR) 1.54, 95\% confidence interval $(\mathrm{CI}) 0.92$ to 2.58]. Neither bed rest in hospital nor bed rest at home showed a significant difference in the prevention of miscarriage. There was a higher risk of 
Bed Rest in Pregnancy and Its R elated Complications: Is It Needed?

miscarriage in those women in the bed rest group than in those in the human chorionic gonadotrophin therapy group with no bed rest ( $R R 2.50,95 \% \mathrm{CI} 1.22$ to 5.11 ). It seems that the small number of participants included in these studies is a main factor to make this analysis inconclusive.

\section{Conclusion}

There is insufficient evidence of high quality that supports a policy of bed rest in order to prevent miscarriage in women with confirmed fetal viability and vaginal bleeding in first half of pregnancy.

(Cochrane Rev A bstract. 2006; C2006 The Cochrane Collaboration).

So various studies have proved that bed rest either does not help or just marginal help may be there and now let us see the problems which are created by advising prolonged bed rest to the patient.

\section{Complications of Bed Rest}

- Bed rest in hospital or at home has financial implications for women and their families, and for health care services

- Prolonged bed rest may be associated with complications such as thrombosis, muscle atrophy or bone demineralization

- The reviewers note that a majority of women in the bedrest group said that they would not choose bed rest in future pregnancies. It is associated with psychological trauma tension and lots of invonvenience.

\section{BONE LOSS IN PREGNANCY}

\section{Introduction}

It is well-documented fact that bed rest, even in healthy and nonpregnant patients leads to bone loss, its use has been reduced in conditions of low backacheand myocardial infarction where it is known it is not effective.

\section{Bone Loss Magnitude}

The pattern of calcium imbalance and bone loss due to disuse is similar in prolonged bed rest, immobilization, spinal cord injury and space travel. U rinary cal cium increases within days of the onset of disuse, and the body's calcium balance may become negative, reaching a peak at about 5 weeks (Hangartner, 1995). However, there are differences in magnitude. In bed rest, the average urinary calcium loss at the peak is about 150 mg per day, which corresponds to $0.5 \%$ of total body calcium (Deitrick, 1948; Donaldson, 1970; Hangartner, 1995). L osses in bone density is greatest in weight-bearing bones with a large proportion of trabecular bone, such as the heel bone. The amount of bone loss in the spine is smaller and occurs later; in some studies, no significant bone loss was detectable in the spine (Hangartner, 1995; L eBlanc, 1987).

\section{EFFECT OF PREGNANCY ON BONE LOSS}

Effect of pregnancy on bone metabolism is complex. $M$ ineralization of fetal skeleton and expansion of maternal plasma volume create an increase demand on maternal cal cium sources which is offset by increased absorption from the intestines and perhaps mobilization of calcium from maternal skeleton.

Two critical reasons exist to study bone loss during pregnancy, if it is irreversible bone loss then it would affect risk of future fracture risk. Consequently, understanding the causes of this bone loss would be a help in future in preventing fractures. Secondly, as a period of heightened bone activity pregnancy is uniquely valuably period for gaining fundamental insight into the detriments of bone processes with possible implications for metabolic bone disorders.

$V$ arious studies have been done to find out the effect of bed rest in pregnancy in producing bone loss. Largest prospective study was done at M agee Women's Hospital and its auxiliary clinics in Pittsburgh and Pensylvania between 1992 and 1995 and 181 healthy pregnant women were enrolled in this study. (AJOG -2004, 191, 1077-83) BMD (bone mass density) was measured at 16 and 36 weeks gestation, i.e. at an interval of 20 weeks. Using DEXA studies at proximal third, middle third and distal third of radius and ulna were done for changes in BMD in the cortical and trabecular bone. Broad array of factors which were taken into account were, bed rest, weight gain during this period, diet, calcium intake, reproductive history, age, $\mathrm{H} / \mathrm{O}$ OCP usage, smoking, caffeine intake, race, $\mathrm{H} / \mathrm{O}$ previous lactation, tobacco use and regular exercise.

B aseline B M D studies were done at 16 w eeks. Evaluation of baseline (gestational week 16) BMD yielded the expected associations; ultradistal BMD exhibited a strong positive correlation with prepregnancy B M I and was greater for A fricanA mericans. Having previously delivered or breastfed were each also somew hat predictive of reduced basel ine ul tradistal BM D. Calcium intake, caffeine consumption, and use of oral contraceptives and tobacco were not meaningfully associated with baseline BMD. Similar, but generally weaker, patterns were observed at the mid- and one-third distal sites.

\section{In Multivariable Models, the Exposure Associated with the Greatest Ultradistal Bone Loss was Prescribed Bedrest}

A fter adjustment for race, age, parity, Iactation history, pregnancy weight gain, calcium intake and exercise, women prescribed bed rest, lost an additional 3.1\% (95\% Cl 0.7-5.4) of ultradistal BM D compared with women not receiving such advice. Characteristics more modestly associated with greater percentage ultradistal bone loss were nulliparity, consuming $<1200 \mathrm{mg} /$ day of calcium, not exercising regularly, gaining $<10$ pounds in the 20 weeks between DX A scans and maternal age $>30$ years. Race was not meaningfully associated with ultradistal bone loss. Neither prepregnancy BM I, ever use of oral contraceptives, tobacco use, and caffeine intake was associated with ultradistal bone loss. 


\section{ADVANTAGES OF EXERCISE DURING PREGNANCY}

There are many fold advantages.

\section{Objective}

To estimate the association between regular exercise before and during pregnancy and excessive new born birth weight.

\section{Methods}

U sing data from the Norwegian mother and child cohort study, 36,869 singleton pregnancies lasting at least 37 weeks were included. Information on regular exercise was based on answers from two questionnaires distributed in pregnancy weeks 17 and 30. Linkage to the Medical Birth Registry of Norway provided data on newborn birth weight.

The main outcome measure was excessive newborn birth weight, defined as birth weight at or above the 90th percentile. Logistic regression analyses were used to estimate the associations separately for nulliparous $(n=16,064)$ and multiparous ( $n=20,805$ ) women, and the results are presented as adjusted odds ratios (aO R s with $95 \%$ confidence intervals).

\section{Results}

Excessivenewborn birth weight was observed in 4,033 (10.9\%) newborns, $56.1 \%(n=2,263)$ of whom were born to multiparous women. An inverse association between regular exercise (at least three times per week) and excessive newborn birth weight in pregnancy weeks 17 and 30 was observed in nulliparous women, aOR $0.72(95 \% \mathrm{Cl} 0.56-0.93)$ and aOR $0.77(95 \% \mathrm{Cl}$ $0.61-0.96)$, respectively. Regular exercise performed before pregnancy did not affect the probability of delivering newborns with an excessive birth weight in nulliparous or multiparous women. Conclusion: Regular exercise during pregnancy reduces the odds of giving birth to newborns with excessive birth weight by 23 to $28 \%$.

OWE KM, Nystad W, Bo K A ssociation between regular exercise and excessive newborn birth weight. O bstet $\mathrm{G}$ ynecol. 2009;114:770-76.

\section{CONCLUSION}

During pregnancy, bed rest is widely used to prevent or treat potential miscarriage, preterm labor, edema, fetal growth retardation, hypertension and pre-eclampsia. There is little evidence of efficacy in improving these pregnancy outcomes, and prescribed bed rest involves substantial emotional, physical and financial costs. ${ }^{2,3} \mathrm{Few}$ randomized trials have been conducted; these have not shown a beneficial effect of bed rest for threatened miscarriage, ${ }^{4}$ hypertension ${ }^{5,6}$ or fetal growth retardation. ${ }^{7}$
The significantly increased ultradistal bone loss associated with bed rest in this study underscores the need for further evaluation of this treatment for specific pregnancy ${ }^{8}$ complications. It is observed, dietary calcium intakes of $2000 \mathrm{mg} /$ day to be modestly protective against ultradistal bone loss. Some routine of exercises must be explained to the patients when in bed.

Exercises should be prescribed for the arms like with resistance bands so that women can carry their babies later on. For the lower limbs rotation at the ankles from $100^{\prime}$ clock to 2PM position should be done along with flexion exercises at the knees.

Stress should be laid down on proper weight gain during this period.

In the postnatal period, weight-bearing exercise should be started as early as possible.

M ore attention should be paid to the nulliparous and women in younger age group.

\section{REFERENCES}

1. A ustralian Institute of Health and W elfare, $\mathrm{N}$ ational Perinatal Statistics U nit. A ustralia's mothers and babies 2000. Canberra. 2003.

2. G oldenberg RL, Cliver SP, B ronstein J, Cutter G, A ndrews W W, $M$ ennemeyer ST. Bed rest in pregnancy. Obstet Gynecol 1994;84:131-36.

3. A llen C, Glasziou P, D el M ar C. B ed rest: A potential ly harmful treatment needing more careful evaluation. Lancet 1999;354:1229-33.

4. Harrison RF. A comparative study of human chorionic gonadotropin, placebo and bed rest for women with early threatened abortion. Int J Fertil Menopausal Stud 1993;38: 160-65.

5. M athews DD, A garwal V, Shuttleworth TP. A randomized controlled trial of complete bed rest versus ambulation in the management of proteinuric hypertension during pregnancy. $\mathrm{Br}$ J Obstet Gynaecol 1982;89:128-31.

6. Tuffnell DJ, Lilford RJ, Buchan PC, Prendiville VM, Tuffnell A , Holgate M P, et al. Randomised controlled trial of day care for hypertension in pregnancy. Lancet 1992;339:224-27.

7. L aurin J, Persson PH, Polberger S. Perinatal outcome in growth retarded pregnancies dated by ultrasound. A cta O bstet Gynecol Scand 1987;66:337-43.

8. Hangartner TN. Osteoporosis due to disuse. In: Matkovic V (Ed), Physical Medicine and Rehabilitation Clinics of North A merica: Osteoporosis. Philadelphia: W B Saunders Company 1995 A ug;6(3):579-94.

\section{ABOUT THE AUTHOR}

\section{Maninder Ahuja}

Director, A huja Hospital and Infertility Center, V isiting Consultant A sian Institute of M edical Sciences, 526, Sector-17, Faridabad Haryana-121002, India, e-mail: ahuja.maninder@gmail.com 